\title{
TOPOLOGICAL AND MULTI-TOPOLOGICAL FRAMES IN THE CONTEXT OF INTUITIONISTIC MODAL LOGIC
}

\begin{abstract}
We present three examples of topological semantics for intuitionistic modal logic with one modal operator $\square$. We show that it is possible to treat neighborhood models, introduced earlier, as topological or multi-topological. From the neighborhood point of view, our method is based on differences between properties of minimal and maximal neighborhoods. Also we propose transformation of multitopological spaces into the neighborhood structures.

Keywords: intuitionistic modal logic, neighbourhood semantics, topological semantics, Kripke frames, soundness and completeness.

Mathematics Subject Classification (2010): 03B45, 03B20, 54A05, 54A10.
\end{abstract}

\section{Introduction}

Neighborhood semantics for intuitionistic propositional logic has been presented by Moniri and Maleki in [9]. Not surprisingly, it turned to be quite similar to the neighborhood semantics for classical modal logic S4. Moreover, the above-mentioned authors proved that their structures correspond to the well-known relational (Kripke) models for intuitionism. It seems that later they became interested rather in neighborhood semantics for subintuitionistic systems (see [7] and [8]).

Nonetheless, even in the context of relatively strong logic like intuitionism, neighborhoods still can provoke certain intuitions. For instance, 
Moniri and Maleki spoke about minimal neighborhoods (which can be identified with upper closed sets in Kripke frames). Hence, it is quite natural to ask also about maximal neighborhoods, i.e. to deny superset axiom. Informally speaking, in this way a place is created for modality. We can assume that necessity means satisfiability in maximal neighborhood.

This assumption led us (see [14]) to the intuitionistic logic with one modal operator $\square$, axiomatized by the rule of necessity and two axioms ( $K$ and $T$ ). Such system has been investigated by Božic and Došen in [1] but with reference to the bi-relational frames. As we have shown, there is a strict correspondence between their setting and our neighborhood approach.

It is well-known (see [10] for more detailed survey) that neighborhood frames for S4 logic behave just like topological structures. This adequacy is true also for intuitionistic neighborhood frames, as it was proved in [9]. For this reason, it is reasonable to look for analogous results for modal logics based on intuitionism. Even if our frames can be presented as bi-relational, we still believe that neighborhoods give us better topological intuitions. In addition, they can be useful when speaking about certain generalizations of topology for weak modal logics (see [13] for details).

Topological semantics for (normal) intuitionistic modal logics has been investigated by Davoren in [4], [5] and Davoren et al. in [6]. Those authors referred to the bi-relational structures with Fischer-Servi conditions (which are not satisfied in our framework). They use specific binary relations between points of topological space. Our idea is different: we do not use any special relation. We limit ourselves to some basic notions like topological neighborhood or open set.

Another concept has been developed by Collinson et al. in [3]. It is based on the notion of topological p-morphism. These authors started from the relational structures and they used some methods of category theory. As for the topological p-morphism, we do not use this tool in the present work. However, we adapted it to the case of generalized topologies in [13].

In [11] we can find some considerations about neighborhood, topological and relational frames for intuitionistic systems with modality. Sotirov assumed that his topological spaces should be equipped with two operations. One of them behaves like interior and is responsible for the intuitionistic features of the logic in question. The second is used to model necessity.

In this research we present different approach. Our first intuition was that neighborhood systems assigned to the particular worlds (i.e. sys- 
tems consisting of minimal and maximal neighborhood) behave like distinct topological spaces in a kind of "meta-universe". We show initial conclusions of this observation. However, in some cases it is better to assume that all these systems are in fact subspaces of one topological space. Hence, we can use the notion of induced topology.

We concentrate only on the basic features of structures mentioned above. In particular, we do not obtain topological completeness because our translations between neighborhood structures (for which we have completeness) and topological spaces (which are defined in three slightly different ways) are one-way. Thus, this paper can be considered as a first step in further studies.

\section{Alphabet and language}

Our basic system is named IKT $\square$. It has rather standard syntax (i.e. alphabet and language). We use the following notations:

1. $P V$ is a fixed denumerable set of propositional variables $p, q, r, s, \ldots$

2. Logical connectives and operators are $\wedge, \vee, \rightarrow, \perp, \square$.

3. The only derived connective is $\neg$ (which means that $\neg \varphi$ is a shortcut for $\varphi \rightarrow \perp$ ).

Formulas are generated recursively in a standard manner: if $\varphi, \psi$ are $w f f$ 's then also $\varphi \vee \psi, \varphi \wedge \psi, \varphi \rightarrow \psi$ and $\square \varphi$. Semantic interpretation of propositional variables and all the connectives introduced above will be presented in the next section. Attention: $\Leftarrow, \Rightarrow$ and $\Leftrightarrow$ are used only on the level of (classical) meta-language.

\section{Neighborhood semantics}

\subsection{The definition of structure}

Our basic structure is an intuitionistic neighborhood modal frame (n2 frame) defined as it follows:

Definition 3.1. $\mathbf{n} 2$-frame is an ordered pair $\langle W, \mathcal{N}\rangle$ where:

1. $W$ is a non-empty set (of worlds, states or points)

2. $\mathcal{N}$ is a function from $W$ into $P(P(W))$ such that: 
(a) $w \in \bigcap \mathcal{N}_{w}$

(b) $\bigcap \mathcal{N}_{w} \in \mathcal{N}_{w}$

(c) $u \in \bigcap \mathcal{N}_{w} \Rightarrow \bigcap \mathcal{N}_{u} \subseteq \bigcap \mathcal{N}_{w}(\rightarrow$-condition $)$

(d) $X \subseteq \cup \mathcal{N}_{w}$ and $\bigcap \mathcal{N}_{w} \subseteq X \Rightarrow X \in \mathcal{N}_{w}$ (relativized superset axiom)

(e) $u \in \bigcap \mathcal{N}_{w} \Rightarrow \bigcup \mathcal{N}_{u} \subseteq \cup \mathcal{N}_{w}$ ( $\square$-condition)

(f) $v \in \bigcup \mathcal{N}_{w} \Rightarrow \bigcap \mathcal{N}_{v} \subseteq \bigcup \mathcal{N}_{w}$ (t-condition)

The first three conditions are in fact taken from pure intuitionism and refer to the features of partial order in relational frames. For instance, $\rightarrow$-condition guarantees that forcing of implication is monotone. As for the relativized superset axiom, it creates place for modality. $\square$-condition is necessary to assure that forcing of modal formulas is also monotone. Significance of the last restriction will be pointed out later.

\subsection{Valuation and model}

DeFinition 3.2. Neighborhood $\mathbf{n} 2$-model is a triple $F_{\mathcal{N}}=\left\langle W, \mathcal{N}, V_{\mathcal{N}}\right\rangle$, where $\langle W, \mathcal{N}\rangle$ is an $\mathbf{n} \mathbf{2}$-frame and $V_{\mathcal{N}}$ is a function from $P V$ into $P(W)$ satisfying the following condition: if $w \in V_{\mathcal{N}}(q)$ then $\bigcap \mathcal{N}_{w} \subseteq V_{\mathcal{N}}(q)$.

Definition 3.3. For every $\mathbf{n} 2$-model $M_{\mathcal{N}}=\left\langle W, \mathcal{N}, V_{\mathcal{N}}\right\rangle$, forcing of formulas in a world $w \in W$ is defined inductively:

1. $w \nVdash \perp$

2. $w \Vdash q \Leftrightarrow w \in V_{\mathcal{N}}(q)$ for any $q \in P V$

3. $w \Vdash \varphi \vee \psi \Leftrightarrow w \Vdash \varphi$ or $w \Vdash \psi$

4. $w \Vdash \varphi \wedge \psi \Leftrightarrow w \Vdash \varphi$ and $w \Vdash \psi$

5. $w \Vdash \varphi \rightarrow \psi \Leftrightarrow \bigcap \mathcal{N}_{w} \subseteq\{v \in W ; v \nVdash \varphi$ or $v \Vdash \psi\}$

6. $w \Vdash \square \varphi \Leftrightarrow \bigcup \mathcal{N}_{w} \subseteq\{v \in W ; v \Vdash \varphi\}$.

As we said, $\neg \varphi$ is a shortcut for $\varphi \rightarrow \perp$. Thus, $w \Vdash \neg \varphi \Leftrightarrow \bigcap \mathcal{N}_{w} \subseteq$ $\{v \in W ; v \nVdash \varphi\}$.

As usual, we say that formula $\varphi$ is satisfied in a model $M_{\mathcal{N}}=\left\langle W, \mathcal{N}, V_{\mathcal{N}}\right\rangle$ when $w \Vdash \varphi$ for every $w \in W$. It is true (tautology) when it is satisfied in each n2-model. 


\section{Neigborhood completeness}

In [14] we have shown (using slightly different symbols) that $\mathbf{n} \mathbf{2}$-frames are sound and complete semantics for the logic IKT $\square$ defined as the following set of formulas and rules: IPC $\cup\{K, T, R N, M P\}$, where:

1. IPC is the set of all intuitionistic axiom schemes

2. $K$ is the axiom scheme $\square(\varphi \rightarrow \psi) \rightarrow(\square \varphi \rightarrow \square \psi)$

3. $T$ is the axiom scheme $\square \varphi \rightarrow \varphi$

4. $R N$ is the rule of necessity: $\varphi \vdash \square \varphi$

5. MP is modus ponens: $\varphi, \varphi \rightarrow \psi \vdash \psi$

Completeness result has been established in two ways. First, directly - by means of prime theories and canonical model. Second, indirectly by the transformation into certain class of bi-relational frames, introduced by Božić and Došen in [1] who proved its completeness. Basically, they used different set of axioms.

\section{Multi-topological frames}

\subsection{The definition of structure and model}

In this section we introduce the notion of multi-topological frame (model). Such structure can be roughly described as a collection of topological spaces with one valuation based on open sets. Each space has its distinguished open set which plays crucial role in the proof of translation between neighborhood and multi-topological settings.

Definition 5.1. mtD-model with distinguished sets is an ordered triple $M_{t}=\left\langle W, \mathfrak{W}, V_{t}\right\rangle$ where:

1. $W \neq \emptyset$.

2. $\mathfrak{W}=\left\{\left\langle T, \tau, D^{\tau}\right\rangle: T \subseteq W, \tau\right.$ is a topology on $\left.T, D^{\tau} \in \tau, D^{\tau} \neq \emptyset\right\}$

3. $W=\bigcup \mathcal{T}$, where $\mathcal{T}=\left\{T:\left\langle T, \tau, D^{\tau}\right\rangle \in \mathfrak{W}\right\}$.

4. $V_{t}$ is a function from $P V$ into $P(W)$ satisfying the following condition: $V_{t}(q)=\bigcup \mathcal{X}$ where $\mathcal{X} \subseteq\left\{X \subseteq W\right.$; there is $\left\langle T, \tau, D^{\tau}\right\rangle \in \mathfrak{W}$ for which $X \in \tau\}$.

The third condition can be formulated also as follows: for each $w \in W$ there is $\left\langle T, \tau, D^{\tau}\right\rangle \in \mathfrak{W}$ such that $w \in T$. Hence, each point of $W$ is at 
least in one topological space. We can consider the whole structure as a universe with many generalized topologies ${ }^{1}$.

For convenience, we shall often identify each $\left\langle T, \tau, D^{\tau}\right\rangle$ simply with $\tau$. As for the valuation of complex formulas, it is based on the valuation of propositional variables and defined inductively:

Definition 5.2. For every $\mathbf{m t D}$-model $M_{t}=\left\langle W, \mathfrak{W}, V_{t}\right\rangle$, valuation of formulas is defined as such:

1. $V_{t}(\varphi \wedge \psi)=V_{t}(\varphi) \cap V_{t}(\psi)$

2. $V_{t}(\varphi \vee \psi)=V_{t}(\varphi) \cup V_{t}(\psi)$

3. $V_{t}(\varphi \rightarrow \psi)=\bigcup_{\tau} \operatorname{Int}_{\tau}\left(-V_{t}(\varphi) \cup V_{t}(\psi)\right)$

4. $V_{t}(\square \varphi)=\bigcup \mathcal{X}$ where $\mathcal{X}=\left\{X \subseteq W\right.$ such that $X=D^{\tau}$ for at least one $\tau$ in $\mathfrak{W}$ such that $\left.T \subseteq V_{t}(\varphi)\right\}$.

A few words of comment should be made. We assume that $V_{t}(q)$ is a union of sets which are open at least in one topology. Concerning value of implication, we look for $-V_{t}(\varphi) \cup V_{t}(\psi)$ and then we sum up all $\tau$-interiors of this set. The last important thing is modality: we check which universes are wholly contained in $V_{t}(\varphi)$ and then we take union of their distinguished sets. We say that formula $\varphi$ is true iff in each $\mathbf{m t D}$-model $M_{t}=\left\langle W, \mathfrak{W}, V_{t}\right\rangle$ we have $V_{t}(\varphi)=W$.

This class of models is based on the observation described above: that we have multiverse of spaces. However, our definition of forcing appears to be too weak (even if we assumed that valuation is based on unions of $\tau$-open sets). Hence, $\mathbf{m t D}$-structures in their most general form are not sound with respect to intuitionism. We did not develop detailed hypothesis about the logic determined by this class of frames. Certainly, some very basic axioms hold. Among them there are: $\varphi \rightarrow \varphi, \varphi \wedge \psi \rightarrow \psi, \varphi \rightarrow \varphi \vee \psi$ and $\psi \rightarrow \varphi \vee \psi$. Also $\varphi \rightarrow(\psi \rightarrow \psi)$ is true. Let us check this fact. Assume that there is a model with a world $w$ such that $w \nVdash \varphi \rightarrow(\psi \rightarrow \psi)$. It means that for each $\tau, w \notin \operatorname{Int}_{\tau}\left(-V_{t}(\varphi) \cup\left(-V_{t}(\psi) \cup V_{t}(\varphi)\right)\right)$. However, the whole expression in brackets is just $W \cup-V_{t}(\psi)=W$. When we take $\tau$-interior of $W$, we obtain subset $T$. Hence, $w$ is beyond any $T$. But this is contradiction.

On the other hand, it is possible that $x \nVdash(\varphi \rightarrow \psi \wedge \psi \rightarrow \gamma) \rightarrow(\varphi \rightarrow \gamma)$, i.e. for each $\tau, x \notin \operatorname{Int}_{\tau}\left(-\left(-V_{t}(\varphi) \cup V_{t}(\psi)\right) \cap\left(-V_{t}(\psi) \cup V_{t}(\gamma)\right)\right) \cup\left(-V_{t}(\varphi) \cup\right.$

\footnotetext{
tions.

${ }^{1}$ Generalized in the sense of Császár (see [2]) but with closure under finite intersec-
} 
$\left.V_{t}(\gamma)\right)$. After some computations the whole expression can be written as $-W \cup\left(-V_{t}(\varphi) \cup V_{t}(\gamma)\right)=-V_{t}(\varphi) \cup V_{t}(\gamma)$. Now take $W=\{w, v, u, z\}$, $\tau_{1}=\{\emptyset,\{w, v\}\}, \tau_{2}=\{\emptyset,\{u, z\}\}, V_{t}(\varphi)=\{w, v\}$, distinguished sets are arbitrary, $V_{t}(\varphi)=\{u, z\}, V_{t}(\psi)=V_{t}(\gamma)=\emptyset$. Now $v$ does not force the formula in question. Let us check it: $\operatorname{Int}_{\tau_{1}}\left(-V_{t}(\varphi) \cup V_{t}(\gamma)\right)=\operatorname{Int}_{\tau_{1}}(\{u, z\})=$ $\emptyset \not \supset v$. Moreover, $\operatorname{Int}_{\tau_{2}}\left(-V_{t}(\varphi) \cup V_{t}(\gamma)\right)=\{u, z\} \not \supset v$.

Also we can easily build a counter-model where $V_{t}(\varphi \wedge \psi) \nsubseteq V_{t}(\top \rightarrow$ $\varphi \wedge \psi)^{2}$.

As for the modal formulas: we can easily prove that axiom $T$ (i.e. $\square \varphi \rightarrow \varphi)$ is always true. Assume that there is a model with $w$ such that $w \nVdash T$. Hence, for any $\tau, w \notin \operatorname{Int}_{\tau}\left(-V_{t}(\square \varphi) \cup V_{t}(\varphi)\right)=\operatorname{Int}_{\tau}(-\bigcup \mathcal{X} \cup$ $V_{t}(\varphi)$ ), where $\mathcal{X}=\left\{X \subseteq W\right.$ such that $X=D^{\tau}$ for at least one $\tau$ in $\mathfrak{W}$ such that $\left.T \subseteq V_{t}(\varphi)\right\}$. Clearly, $\bigcup \mathcal{X} \subseteq V_{t}(\varphi)$. Hence, $-V_{t}(\varphi) \subseteq-\bigcup \mathcal{X}$ which gives us that $-\bigcup \mathcal{X} \cup V_{t}(\varphi)=W$. Again, we obtain impossible result that $w \notin T$ for any $\left\langle T, \tau, D^{\tau}\right\rangle \in \mathfrak{W}$.

On the other hand, axiom 4 (i.e. $\square \varphi \rightarrow \square \square \varphi$ ) can be falsified. Take $W=\{w, v, u\}, \tau_{1}=\{\emptyset,\{w, v\},\{w, v, u\}\}, D^{\tau_{1}}=\{w, v\}, \tau_{2}=\{\emptyset,\{v\}\}$, $D^{\tau_{2}}=\{v\}, V_{t}(\varphi)=W$. Now $V_{t}(\square \varphi)=D^{\tau_{1}} \cup D^{\tau_{2}}=\{w, v\}, V(\square \square \varphi)=$ $D^{\tau_{2}}=\{v\},-V_{t}(\square \varphi) \cup V_{t}(\square \square \varphi)=\{u, v\}$. Hence, $\operatorname{Int}_{\tau_{1}}(\{u, v\})=\emptyset$, Int $_{\tau_{2}}(\{u, v\})=\{v\}$. Clearly, $u$ is beyond those interiors, so $u \nVdash 4$.

We see that the logic of mtD -frames is a kind of unknown subintuitionistic modal logic. We conjecture that it may be fruitful to study general multi-topological structures and to look for any regularities depending on mutual location of spaces or their topological properties. We signalize this possibility but it is beyond the scope of present paper. And so, overall here, we shall work only with a certain subclass of these structures, namely i-mtD-frames.

Definition 5.3. We say that $\mathbf{m t} \mathbf{D}$-frame is $\mathbf{i}-\mathbf{m t D}$ iff there is an Alexandrov topology $\mu$ on $W$ such that for each $\tau \in \mathfrak{W}, \tau$ is a subspace topology induced by $\mu$.

If we speak about Alexandrov topology, it means that arbitrary intersections of open sets are also open. If $\tau$ on $T$ is induced by $\mu$, then each $U \in \tau$ can be presented as $T \cap A$ for certain $A \in \mu$. On the other hand, if $A \in \mu$, then $T \cap A \in \tau$. This subclass of models is sound with respect to

\footnotetext{
${ }^{2}$ We are grateful to the anonymous reviewer for this example and some other important comments.
} 
intuitionism what can be manually checked. It is well-known fact that subspaces of Alexandrov space also have Alexandrov property (see Theorem 7 in $[12])$.

\section{From neighborhood frames to multi-topological structures}

\subsection{Basic notions}

In this section we show that it is possible to treat neighborhood models as multi-topological. First, let us introduce the notion of $w$-open sets.

Definition 6.1. We say that set $X \subseteq W$ is $w$-open in $\mathbf{n 2 - f r a m e ~ i f f ~} X \subseteq$ $\bigcup \mathcal{N}_{w}$ and for every $v \in X$ we have $\bigcap \mathcal{N}_{v} \subseteq X$. We define $\mathcal{O}_{w}$ as $\{X \subseteq$ $W: X$ are $w$-open $\}$ and call it $w$-topology.

Let us check that this definition is useful for our needs.

Theorem 6.2. Assume that we have $\mathbf{n} 2$-frame $F_{\mathcal{N}}=\langle W, \mathcal{N}\rangle$. Then $\mathcal{O}_{w}$ is a topological space for every $w \in W$.

Proof: Let us check standard properties of topology.

1. Take empty set. We can say that $\emptyset \in \mathcal{O}_{w}$ because $\emptyset \subseteq \cup \mathcal{N}_{w}$ and there are no any $v$ in $\emptyset$.

2. Consider $\bigcup \mathcal{N}_{w}$. Clearly this set is contained in itself and because of $T$-condition we have that for every $v \in \cup \mathcal{N}_{w}$ the second condition holds: $\bigcap \mathcal{N}_{v} \subseteq \cup \mathcal{N}_{w}$.

3. Consider $\mathscr{X} \subseteq \mathcal{O}_{w}$. We show that $\bigcap \mathscr{X} \in \mathcal{O}_{w}$. The first condition is simple: every element of $\mathscr{X}$ belongs to $\mathcal{O}_{w}$ so it is contained in $\bigcup \mathcal{N}_{w}$. The same holds of course for intersection of all such elements. Now let $v \in \bigcap \mathscr{X}$. By the definition we have that $\bigcap \mathcal{N}_{v} \subseteq X$ for every $X \in \mathscr{X}$. Then $\bigcap \mathcal{N}_{v} \subseteq \bigcap \mathscr{X}$.

4. In the last case we deal with arbitrary unions. Suppose that $\mathscr{X} \subseteq \mathcal{O}_{w}$ and consider $\bigcup \mathscr{X}$. Surely this union is contained in $\bigcup \mathcal{N}_{w}$. Now let us take an arbitrary $v \in \bigcup \mathscr{X}$. We know that $\bigcap \mathcal{N}_{v} \subseteq X$ for some $X \in \mathscr{X}$ (in fact, it holds for every $X$ which contains $v$ ). Then clearly $\bigcap \mathcal{N}_{v} \subseteq \bigcup \mathscr{X}$.

One thing should be noted. Clearly, we used $t$-condition to assure that the whole maximal $w$-neighborhood is $w$-open. Basically, in [14], we worked 
with structures without $t$-condition (we may call them $\mathbf{n} \mathbf{1}$-frames). Completeness theorem holds also for them - but it would be at least problematic to treat those frames as multi-topological.

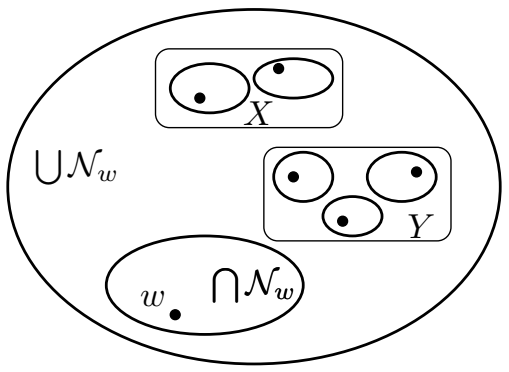

Fig. 1. Topology $O_{w}$. X, Y are $w$-open.

Theorem 6.3. Assume that we have $M_{\mathcal{N}}=\left\langle W, \mathcal{N}, V_{\mathcal{N}}\right\rangle$ and we define topology $\mu$ on $W$ in the following way: if $A \subseteq W$, then $A \in \mu \Leftrightarrow$ for any $v \in A, \bigcap \mathcal{N}_{v} \subseteq A$. Then, for any $w \in W, \mathcal{O}_{w}$ is induced by $\mu$ (i.e. $\mathcal{O}_{w}$ is subspace topology).

Proof: Let us take $w \in W$. We shall prove that $\mathcal{O}_{w}$ consists strictly of intersections of $\bigcup \mathcal{N}_{w}$ and $\tau$-open sets.

If $U \in \mathcal{O}_{w}$ then $U \in \mu$ (this is clear) and $U=U \cap \bigcup \mathcal{N}_{w}$. Assume now that $A$ is $\mu$-open and consider $Z=A \cap \bigcup \mathcal{N}_{w}$. Let us check that this set belongs to $\mathcal{O}_{w}$. Of course it is contained in $\bigcup \mathcal{N}_{w}$. Suppose that there is $z \in Z$ such that $\bigcap \mathcal{N}_{z} \nsubseteq Z$. But $\bigcap \mathcal{N}_{z} \subseteq A$ (because $A$ is $\tau$-open) and $\bigcap \mathcal{N}_{z} \subseteq \cup \mathcal{N}_{w}$ (because of $t$-condition). This is contradiction.

Additionally, one can easily check that $\mu$ is Alexandrov.

\subsection{Transformation}

Theorem 6.4. For each $\mathbf{n} 2$-model $M_{\mathcal{N}}=\left\langle W, \mathcal{N}, V_{\mathcal{N}}\right\rangle$ there exists i-mtD model $M_{t}=\left\langle W, \mathfrak{W}, V_{t}\right\rangle$ which is pointwise equivalent to $M_{\mathcal{N}}$, i.e. $w \Vdash \varphi \Leftrightarrow$ $w \in V_{t}(\varphi)$.

Proof: Assume that we have $M_{\mathcal{N}}=\left\langle W, \mathcal{N}, V_{\mathcal{N}}\right\rangle$. Now let us consider the following structure: $M_{t}=\left\langle W, \mathfrak{W}, V_{t}\right\rangle$ where:

1. $\mathfrak{W}=\left\{\left\langle\bigcup \mathcal{N}_{w}, \mathcal{O}_{w}, \bigcap \mathcal{N}_{w}\right\rangle ; w \in W\right\}$

2. for each $q \in P V, V_{t}(q)=V_{\mathcal{N}}(q)$ 
We shall identify each $\left\langle\bigcup \mathcal{N}_{w}, \mathcal{O}_{w}, \bigcap \mathcal{N}_{w}\right\rangle$ just with $\mathcal{O}_{w}$. It is easy to check that this is well-defined i-mtD-frame. For each $w \in W$ we can treat $\bigcup \mathcal{N}_{w}$ as a universe of topological subspace. Thus $\bigcap \mathcal{N}_{w}$ can be treated as distinguished set in this particular subspace.
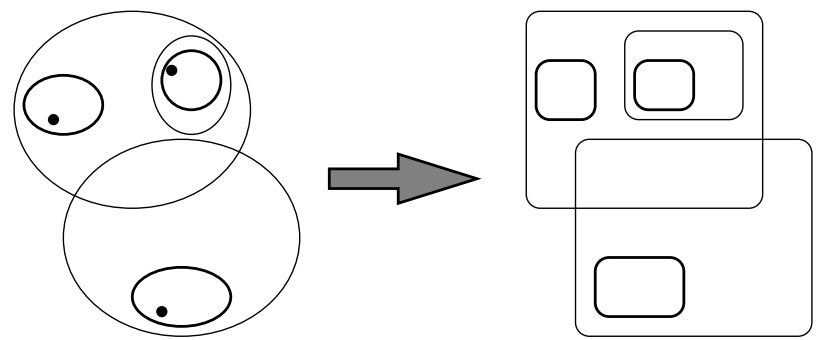

Fig. 2. From neighborhoods to multi-topological space with distinguished sets.

Now let us prove pointwise equivalency. Here we use induction by the complexity of formulas.

1. $\rightarrow$ :

$(\Rightarrow)$ Suppose that $w \Vdash \varphi \rightarrow \psi$. We want to show there exists certain $\left\langle\bigcup \mathcal{N}_{x}, \mathcal{O}_{x}, \bigcap \mathcal{N}_{x}\right\rangle \in \mathfrak{W}$ such that $w \in \operatorname{Int}_{x}\left(\left(-V_{t}(\varphi) \cup V_{t}(\psi)\right)\right.$.

We can say that $w \in \bigcap \mathcal{N}_{w} \subseteq\{x \in W ; x \nVdash \varphi$ or $x \Vdash \psi\}$. By induction hypothesis, this set can be written as $\left\{x \in W ; x \notin V_{t}(\varphi)\right.$ or $\left.x \in V_{t}(\psi)\right\}=-V_{t}(\varphi) \cup V_{t}(\psi)$. Recall the fact that $\bigcap \mathcal{N}_{w} \subseteq \cup \mathcal{N}_{w}$. Thus $w \in \bigcap \mathcal{N}_{w} \subseteq\left(-V_{t}(\varphi) \cup V_{t}(\psi)\right) \cap \bigcup \mathcal{N}_{w}$. But $\bigcap \mathcal{N}_{w}$ is $w$-open so it is contained in $\operatorname{Int}_{w}\left(-V_{t}(\varphi) \cup V_{t}(\psi)\right)$. We see that we could treat $w$ as our $x$.

$(\Leftarrow)$ Now we assume that $w \in V_{t}(\varphi \rightarrow \psi)$. Thus we have certain $\mathcal{O}_{x}$ such that $w \in \operatorname{Int}_{x}\left(\left(-V_{t}(\varphi) \cap V_{t}(\psi)\right)\right.$. By induction hypothesis, $w \in \operatorname{Int}_{x}(\{z \in W ; z \nVdash \varphi$ or $z \Vdash \psi\})$. Hence, $w$ belongs to the biggest $x$-open set $X$ such that $X \subseteq\{z \in W ; z \nVdash \varphi$ or $z \Vdash \psi\}$. But if $X$ is $x$-open then $\bigcap \mathcal{N}_{w} \subseteq X$. Thus $w \Vdash \varphi \rightarrow \psi$.

2. $\square$ :

$(\Rightarrow)$ Assume that $w \Vdash \square \varphi$. We want to show that $w \in V_{t}(\square \varphi)$, i.e. that there is $X \subseteq W$ such that $w \in X$ and for certain $\mathcal{O}_{x}$ we have: $X=\bigcap \mathcal{N}_{x}, \bigcup \mathcal{N}_{x} \subseteq V_{t}(\varphi)$. 
Surely, we can take $x=w$. Now, if $w \Vdash \square \varphi$, then $\bigcup \mathcal{N}_{w} \subseteq V_{\mathcal{N}}(\varphi)$. By induction hypothesis, $\bigcup \mathcal{N}_{w} \subseteq V_{t}(\varphi)$.

$(\Leftarrow)$ Suppose that $w \in V_{t}(\square \varphi)$. Thus $w \in X \subseteq W$ such that for certain $\mathcal{O}_{x}$ we can say that $X=\bigcap \mathcal{N}_{x}$ and $\bigcup \mathcal{N}_{x} \subseteq V_{t}(\varphi)$.

If $\bigcup \mathcal{N}_{s} \subseteq V_{t}(\varphi)$, then (by induction hypothesis) $\bigcup \mathcal{N}_{x} \subseteq V_{\mathcal{N}}(\varphi)$. Thus $x \Vdash \square \varphi$. But $w \in \bigcap \mathcal{N}_{x}$. Thus, by the monotonicity of intuitionistic forcing, $w \Vdash \square \varphi$.

\section{From multi-topological structures to neighborhood structures}

In the former section we used multi-topological structures with distinguished open sets $D^{\tau}$. Those sets are equivalents of minimal $w$-neighborhoods (while subspaces played the role of maximal $w$-neighborhoods). We used such unconventional approach mainly because our topology $\mathcal{O}_{w}$ does not "recognize" minimal neighborhoods. Thus, if we have $\bigcup \mathcal{N}_{w}$, then from the neighborhood point of view $\bigcap \mathcal{N}_{w}$ is specific - but as $w$-open set it is not distinguished in any way from other $w$-open sets. But we need such distinction to establish correspondence between $V_{\mathcal{N}}$ and $V_{t}$.

Now we are on the other side: we start from topological structures but defined in slightly different way. Here we do not have $D^{\tau}$ sets. We have the following definition (of frame):

DeFinition 7.1. t2-frame is an ordered pair $\langle W, \mathfrak{W}\rangle$ where:

1. $W \neq \emptyset$

2. $\mathfrak{W}=\{\langle T, \tau\rangle: T \subseteq W, \tau$ is an Alexandrov topology on $T\}$.

3. $W=\bigcup \mathcal{T}$, where $\mathcal{T}=\{T ;\langle T, \tau\rangle \in \mathfrak{W}\}$

Each $\langle T, \tau\rangle$ is an Alexandrov space, so each $w \in T$ has its minimal $\tau$-open neighborhood. If we denote the family of $\tau$-open $w$-neighborhoods as $\mathcal{O}_{\tau}^{w}$, then we can introduce the following notation: $\bigcap \mathcal{O}_{\tau}^{w}=\min \mathcal{O}_{\tau}^{w}$.

Our definition of frame is very similar to Def. 5.1 but now we deny distinguished sets. However, in the definition of model there are bigger differences. In fact, we shall define forcing after introducing specific kind of neighborhoods in our topological environment.

Now let us think about intersection of all minimal $\tau$-open $w$-neighborhoods. It will be denoted as $\bigcap_{\langle T, \tau\rangle \in \mathcal{T}^{w}}\left\{\min \mathcal{O}_{\tau}^{w}\right\}$ or shortly by $\bigcap_{\tau \in \mathcal{T} w}\left\{\min \mathcal{O}_{\tau}^{w}\right\}$, where $\mathcal{T}^{w}=\{\langle T, \tau\rangle \in \mathfrak{W}: w \in T\}$. Below we define neighborhoods in the sense mentioned above. 
Definition 7.2. Assume that we have t2-frame $\langle W, \mathfrak{W}\rangle$. Then for each $w \in W$ we define:

1. $\bigcap \mathcal{N}_{w}^{t}=\bigcap_{\tau \in \mathcal{T} w}\left\{\min \mathcal{O}_{\tau}^{w}\right\}$

2. $\bigcup \mathcal{N}_{w}^{t}=\bigcap \mathcal{T}^{w}$

3. $X \in \mathcal{N}_{w}^{t} \subseteq P(P(W)) \Leftrightarrow \bigcap \mathcal{N}_{w}^{t} \subseteq X \subseteq \bigcup \mathcal{N}_{w}^{t}$

TheOREM 7.3. Assume that we have $\mathbf{t 2}$-frame $\langle W, \mathfrak{W}\rangle$ with $\mathcal{N}_{w}^{t}$ defined as in Def. 7.2. We state that for each $w \in U, \mathcal{N}_{w}^{t}$ has all the properties of neighborhood family in $\mathbf{n} \mathbf{2}$-frame.

Proof: We must check five conditions:

1. $w \in \bigcap \mathcal{N}_{w}^{t}$. This is simple because $\bigcap \mathcal{N}_{w}^{t}$ is defined as an intersection of all $\tau$-open $w$-neighborhoods (for every $\tau$ in $\mathcal{T}^{w}$ ) and certainly $w$ is in each such neighborhood.

2. $\bigcap \mathcal{N}_{w}^{t} \in \mathcal{N}_{w}^{t}$. This is obvious by the very definition of $\mathcal{N}_{w}^{t}$.

3. $v \in \bigcap \mathcal{N}_{w}^{t} \Rightarrow \bigcap \mathcal{N}_{v}^{t} \subseteq \bigcap \mathcal{N}_{w}^{t}$. Let us note two facts. First, $v$ is at least in all those spaces, in which $w$ is (because it is in the intersection of all minimal $w$-neighborhoods). Thus, we can say that $\bigcap \mathcal{N}_{v}^{t}=$ $\bigcap_{\tau \in \mathcal{T}^{v}}\left\{\min \mathcal{O}_{\tau}^{v}\right\} \subseteq \bigcap_{\tau \in \mathcal{T}^{w}}\left\{\min \mathcal{O}_{\tau}^{v}\right\}$.

Second, suppose for a moment that we work with one particular Alexandrov topological space $\rho$. Assume that $v$ belongs to the minimal $\rho$-open neighborhood of $w$. Of course $v$ has its own minimal $\rho$-open neighborhood - but let us suppose that $\min \mathcal{O}_{\rho}^{v} \nsubseteq \min \mathcal{O}_{\rho}^{w}$. Now - from the basic properties of topology and the fact that at least $v$ belongs to $\min \mathcal{O}_{\rho}^{w}$ - we state that $\min \mathcal{O}_{\rho}^{v} \cap \min \mathcal{O}_{\rho}^{w}$ is $\rho$-open. Of course, this intersection is contained in $\min \mathcal{O}_{\rho}^{w}$. Thus, we have contradiction with the assumption that minimal $\rho$-open $v$-neighborhood is not contained in $\min \mathcal{O}_{\rho}^{w}$.

Now let us go back to the main part of the proof. The second fact allows us to say that $\bigcap_{\tau \in \mathcal{T} w}\left\{\min \mathcal{O}_{\tau}^{v}\right\} \subseteq \bigcap_{\tau \in \mathcal{T} w}\left\{\min \mathcal{O}_{\tau}^{w}\right\}=\bigcap \mathcal{N}_{w}^{t}$.

4. $v \in \bigcap \mathcal{N}_{w}^{t} \Rightarrow \bigcup \mathcal{N}_{v}^{t} \subseteq \bigcup \mathcal{N}_{w}^{t}$. As earlier, we say that $v$ is at least in each space which belongs to $\mathcal{T}^{w}$. Thus $\bigcup \mathcal{N}_{v}^{t}=\bigcap \mathcal{T}^{v}=\bigcap\{\langle T, \tau\rangle \in$ $\mathfrak{W}: v \in T\} \subseteq \bigcap\{\langle T, \tau\rangle \in \mathfrak{W}: w \in T\}=\bigcup \mathcal{N}_{w}^{t}$.

5. $v \in \bigcup \mathcal{N}_{w}^{t} \Rightarrow \bigcap \mathcal{N}_{v}^{t} \subseteq \bigcup \mathcal{N}_{w}^{t}$. Suppose that $v \in \bigcup \mathcal{N}_{w}^{t}$ defined as in Def. 7.2. Thus $v \in \bigcap \mathcal{T}^{w}$ which means in particular that $v$ is in all those universes, in which $w$ is. Now it is clear that $\bigcap \mathcal{N}_{v}^{t}$ - defined as an intersection of all $\tau$-open minimal $v$-neighborhoods - must be contained at least in each element of $\mathcal{T}^{w}$, i.e. in $\bigcup \mathcal{N}_{w}^{t}$. 


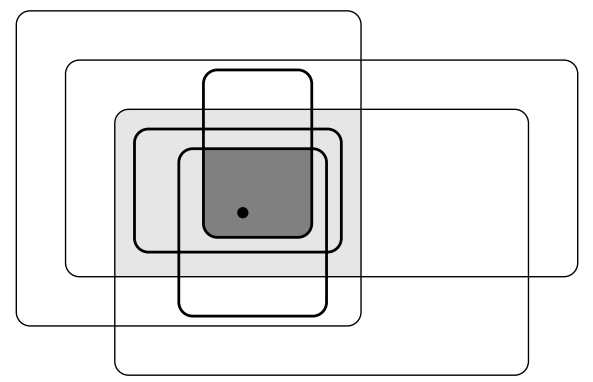

Fig. 3. Maximal and minimal neighborhoods in multi-topological space.

We have transformed our initial multi-topological structure into the neighborhood frame. Note that it is possible that for certain (and even for each) $\tau$ the set $\bigcap \mathcal{N}_{w}^{t}$ is not $\tau$-open. We do not expect this. It is just intersection of all minimal $w$-neighborhoods. Now we shall introduce valuation and rules of forcing - thus obtaining logical model.

Definition 7.4. Assume that we have t2-frame $\langle W, \mathfrak{W}\rangle$. Suppose that for each $w \in W$ we defined $\mathcal{N}_{w}^{t}$ as in Def. 7.2. We define valuation $V_{t}$ as a function from $P V$ into $P(W)$ satisfying the following condition: if $w \in V_{t}(q)$ then $\bigcap \mathcal{N}_{w}^{t} \subseteq V_{t}(q)$. The whole triple $\left\langle W, \mathfrak{W}, V_{t}\right\rangle$ is called t2 model.

Definition 7.5. For every $\mathbf{t 2}$-model $M_{t}=\left\langle W, \mathfrak{W}, V_{t}\right\rangle$, valuation of formulas is defined as such:

1. $V_{t}(\varphi \wedge \psi)=V_{t}(\varphi) \cap V_{t}(\psi)$

2. $V_{t}(\varphi \vee \psi)=V_{t}(\varphi) \cup V_{t}(\psi)$

3. $V_{t}(\varphi \rightarrow \psi)=\bigcup_{x \in \mathscr{I}}\left\{\bigcap \mathcal{N}_{x}^{t}\right\}$ where $\mathscr{I}=\left\{x \in W: \bigcap \mathcal{N}_{x}^{t} \subseteq-V_{t}(\varphi) \cup\right.$ $\left.V_{t}(\psi)\right\}$

4. $V_{t}(\square \varphi)=\bigcup_{x \in \mathscr{M}}\left\{\bigcap \mathcal{N}_{x}^{t}\right\}$ where $\mathscr{M}=\left\{x \in W: \bigcup \mathcal{N}_{x}^{t} \subseteq V_{t}(\varphi)\right\}$

We say that formula $\varphi$ is true iff in each t2-model $M_{t}=\left\langle W, \mathfrak{W}, V_{t}\right\rangle$ we have $V_{t}(\varphi)=W$.

The next theorem is crucial for our considerations.

Theorem 7.6. For each $\mathbf{t 2}$-model $M_{t}=\left\langle W, \mathfrak{W}, V_{t}\right\rangle$ there exists $\mathbf{n} \mathbf{2}$-model $M_{\mathcal{N}}=\left\langle W, \mathcal{N}, V_{\mathcal{N}}\right\rangle$ which is pointwise equivalent to $M_{t}$, i.e. $w \Vdash \varphi \Leftrightarrow w \in$ $V_{t}(\varphi)$.

Proof: Let us take $M_{t}$ and introduce $\mathcal{N}_{w}^{t}$ for each $w \in W$ just like in Def. 7.2. We define $V_{\mathcal{N}}: P V \rightarrow P(W)$ in the following way: $V_{\mathcal{N}}=V_{t}$. 
Now the structure $M_{\mathcal{N}}=\left\langle W, \mathcal{N}^{t}, V_{\mathcal{N}}\right\rangle$ is a proper neighborhood model. In fact, we have already shown that it is $\mathbf{n} \mathbf{2}$-frame. By the definition of $V_{t}$ we know that it is monotone in $\mathbf{n} \mathbf{2}$-frame. Let us check pointwise equivalency between both structures.

1. $\rightarrow$

$(\Rightarrow)$ Suppose that $w \Vdash \varphi \rightarrow \psi$. Thus $\bigcap \mathcal{N}_{w}^{t} \subseteq\{v \in W ; v \nVdash \varphi$ or $v \Vdash \psi\}=-V_{\mathcal{N}}(\varphi) \cup V_{\mathcal{N}}(\psi)$. By induction this last set can be written as $-V_{t}(\varphi) \cup V_{t}(\psi)$. Thus, we can say that $w$ belongs to $\mathscr{I}$ defined as in Def. 7.2. Of course $w \in \bigcap \mathcal{N}_{w}^{t}$. Hence, $w \in V_{t}(\varphi \rightarrow \psi)$.

$(\Leftarrow)$ Assume that $w \in V_{t}(\varphi \rightarrow \psi)$. This means that there is at least one point $x \in \mathscr{I}$ such that $w \in \bigcap \mathcal{N}_{x}^{t}$. But if $\bigcap \mathcal{N}_{x}^{t} \subseteq-V_{t}(\varphi) \cup V_{t}(\psi)$ then we can say that $\bigcap \mathcal{N}_{x}^{t} \subseteq-V_{\mathcal{N}}(\varphi) \cup V_{\mathcal{N}}(\psi)$ (by induction). Hence, $x \Vdash \varphi \rightarrow \psi$. The same can be said about $w$ (because $w \in \bigcap \mathcal{N}_{x}^{t}$ ).

2 .

$(\Rightarrow)$ Suppose that $w \Vdash \square \varphi$. Thus $\bigcup \mathcal{N}_{w} \subseteq V_{\mathcal{N}}(\varphi)=V_{t}(\varphi)$. The last equivalence is a result of induction hypothesis. Now we see that $w \in \mathscr{M}$. Of course $w \in \bigcap \mathcal{N}_{w}^{t}$. Then $w \in V_{t}(\square \varphi)$.

$(\Leftarrow)$ Assume that $w \in V_{t}(\square \varphi)$. Hence, there is at least one world $x \in \mathscr{M}$ such that $w \in \bigcap \mathcal{N}_{x}^{t}$. But if $\bigcup \mathcal{N}_{x}^{t} \subseteq V_{t}(\varphi)$, then by induction $\bigcup \mathcal{N}_{x}^{t} \subseteq V_{\mathcal{N}}(\varphi)$. This means that $x \Vdash \square \varphi$. By monotonicity of forcing in $\bigcap \mathcal{N}_{x}^{t}$ we can say that $w \Vdash \square \varphi$.

\section{Alternative approach}

Let us go to back to the $\mathbf{n} \mathbf{2}$-frames. We shall define topology in a slightly different way than in Def. 6.1. Now we assume that $\bigcap \mathcal{N}_{w}$ is always contained in each $w$-open set.

Definition 8.1. Suppose that we have $\mathbf{n} 2$-frame $M_{\mathcal{N}}=\langle W, \mathcal{N}\rangle$. We say that $X \subseteq W$ is $w_{\text {min }}$-open in $\mathbf{n 2}$-structure iff $X=\emptyset$ or $X \subseteq \cup \mathcal{N}_{w}$, $\bigcap \mathcal{N}_{w} \subseteq X$ and for every $v \in X$ we have $\bigcap \mathcal{N}_{v} \subseteq X$. We denote $\mathcal{Q}_{w}=$ $\left\{X \subseteq W: X\right.$ are $w_{\text {min }}$-open $\} \cup \emptyset$ and call it $w_{\text {min }}$-topology.

Theorem 8.2. Assume that we have $\mathbf{n 2}$-frame $F_{\mathcal{N}}=\langle W, \mathcal{N}\rangle$. Then $\left\langle\bigcup \mathcal{N}_{w}, \mathcal{Q}_{w}\right\rangle$ is a topological space (for every $w \in W$ ).

Proof: It is easy to check conditions of well-defined topology - just as in Th. 6.2. We leave details to the reader. 


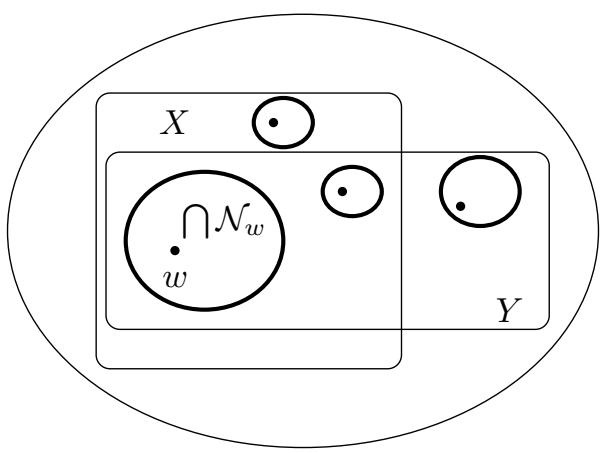

Fig. 4. Topology $Q_{w}$. X, Y are $w$-open.

\subsection{From neighborhood frames to multi-topological structures once again}

Let us introduce the new type of multi-topological structures. In fact, they are $\mathbf{t} 2$-frames but with valuation defined in a different way. Recall that $\mathcal{O}_{\tau}^{w}$ denotes the family of all $\tau$-open $w$-neighborhoods and $\min \mathcal{O}_{\tau}^{w}$ is an intersection of such family.

Definition 8.3. t3-model is an ordered triple $M_{t}=\langle W, \mathfrak{W}, V\rangle$ where $\langle W, \mathfrak{W}\rangle$ is a $\mathbf{t} 2$-frame and $V_{t}$ is a function from $P V$ into $P(W)$ satisfying the following condition: $V_{t}(q)=\bigcup \mathcal{X}$ where $\mathcal{X} \subseteq\{X \subseteq W$; there is $\langle T, \tau\rangle \in \mathfrak{W}$ and $w \in T$ such that $X=\min \mathcal{O}_{\tau}^{w}$ \}.

Definition 8.4. For every $\mathbf{t} 3$-model $M_{t}=\left\langle W, \mathfrak{W}, V_{t}\right\rangle$, valuation of formulas is defined as such:

1. $V_{t}(\varphi \wedge \psi)=V_{t}(\varphi) \cap V_{t}(\psi)$

2. $V_{t}(\varphi \vee \psi)=V_{t}(\varphi) \cup V_{t}(\psi)$

3. $V_{t}(\varphi \rightarrow \psi)=\bigcup \mathcal{X}$, where $\mathcal{X}=\left\{X \subseteq W\right.$ such that $X \subseteq-V_{t}(\varphi) \cup$ $V_{t}(\psi)$ and there are $\langle T, \tau\rangle \in \mathfrak{W}, x \in T$ for which $\left.X=\min \mathcal{O}_{\tau}^{x}\right\}$.

4. $V_{t}(\square \varphi)=\bigcup \mathcal{X}$, where $\mathcal{X}=\{X \subseteq W$ such that there are $\langle T, \tau\rangle \in \mathfrak{W}$, $x \in T$ for which $X=\min \mathcal{O}_{\tau}^{x}$ and $\left.T \subseteq V_{t}(\varphi)\right\}$.

We say that formula $\varphi$ is true iff in each $\mathbf{t 3}$-model $M_{t}=\left\langle W, \mathfrak{W}, V_{t}\right\rangle$ we have $V_{t}(\varphi)=W$. 
One can see that in some sense we composed earlier definitions of multitopological frames, valuations and models. Now our situation is similar to that from section 5 . The main difference is that we can work with minimal $\tau$-open sets, i.e. with $\min \mathcal{O}_{\tau}^{w}$.

TheOREM 8.5. For each $\mathbf{n 2}$-model $M_{\mathcal{N}}=\left\langle W, \mathcal{N}, V_{\mathcal{N}}\right\rangle$ there exists $\mathbf{t 3}$ model $M_{t}=\left\langle W, \mathfrak{W}, V_{t}\right\rangle$ which is pointwise equivalent to $M_{\mathcal{N}}$, i.e. $w \Vdash \varphi \Leftrightarrow$ $w \in V_{t}(\varphi)$.

Proof: Assume that we have $M_{\mathcal{N}}=\left\langle W, \mathcal{N}, V_{\mathcal{N}}\right\rangle$. Now let us consider the following structure: $M_{t}=\left\langle W, \mathfrak{W}, V_{t}\right\rangle$ where:

1. $\mathfrak{W}=\left\{\left\langle\bigcup \mathcal{N}_{w}, \mathcal{Q}_{w}\right\rangle: w \in W\right\}$

2. for each $q \in P V, V_{t}(q)=V_{\mathcal{N}}(q)$

It is easy to check that $\langle W, \mathfrak{W}\rangle$ is a well-defined $\mathbf{t 2}$-frame. Let us prove pointwise equivalency by means of induction.

$\rightarrow$

$(\Rightarrow)$ Suppose that $w \Vdash \varphi \rightarrow \psi$. Thus $\bigcap \mathcal{N}_{w} \subseteq\{v \in W ; v \nVdash \varphi$ or $v \Vdash \psi\}$. The last set - by induction hypothesis - is equal to $-V_{t}(\varphi) \cup V_{t}(\psi)$. Moreover, $\bigcap \mathcal{N}_{w}$ is an intersection of all $w_{\text {min-open sets (recall Def. 8.1) }}$ and $w \in \bigcap \mathcal{N}_{w} \subseteq \bigcup \mathcal{N}_{w}$. Thus $w \in V_{t}(\varphi \rightarrow \psi)$.

$(\Leftarrow)$ Assume that $w \in V_{t}(\varphi \rightarrow \psi)$. First, there is $X \subseteq W$ such that $w \in X$ and $X \subseteq-V_{t}(\varphi) \cup V_{t}(\psi)$. Second, there is $\left\langle\bigcup \mathcal{N}_{x}, \mathcal{Q}_{x}\right\rangle \in \mathfrak{W}$ such that $X$ is minimal $\mathcal{Q}_{x}$-open $x$-neighborhood. In fact, it means that $X=\bigcap \mathcal{N}_{x}$. So $\bigcap \mathcal{N}_{x} \subseteq-V_{t}(\varphi) \cup V_{t}(\psi)=$ [ind. hyp. $]-V_{\mathcal{N}}(\varphi) \cup V_{\mathcal{N}}(\psi)=\{z \in W ; z \nVdash \varphi$ or $z \Vdash \psi\}$. Then, in particular, $x \Vdash \varphi \rightarrow \psi$ and also $w \Vdash \varphi \rightarrow \psi$ (because $w \in \bigcap \mathcal{N}_{x}$ and we have intuitionistic monotonicity of forcing).

$(\Rightarrow)$ Suppose that $w \Vdash \square \varphi$. Thus $\bigcup \mathcal{N}_{w} \subseteq\{v \in W ; v \Vdash \varphi\}$. The last set is - by induction hypothesis - equal to $V_{t}(\varphi)$. We can say that conditions from Def. 8.4 are satisfied: our $X$ is $\bigcap \mathcal{N}_{w}$ and our topological space is $\left\langle\bigcup \mathcal{N}_{w}, \mathcal{Q}_{w}\right\rangle$. Thus $w \in V_{t}(\square \varphi)$.

$(\Leftarrow)$ Assume that $w \in V_{t}(\square \varphi)$. Thus, we have $X \subseteq W$ such that $w \in X$ and there are $x \in W,\left\langle\bigcup \mathcal{N}_{x}, \mathcal{Q}_{x}\right\rangle \in \mathfrak{W}$ such that $X$ is $\bigcap \mathcal{N}_{x}$ (i.e. minimal $\mathcal{Q}_{x}$-open $x$-neighborhood) and $\bigcup \mathcal{N}_{x} \subseteq V_{t}(\varphi)$. By induction hypothesis $\bigcup \mathcal{N}_{x} \subseteq V_{\mathcal{N}}(\varphi)$. Thus, $x \Vdash \square \varphi$. By monotonicity of forcing, $w \Vdash \varphi$. 


\section{Summary}

In this paper we used a lot of notions and symbols. We have introduced three different concepts of multi-topological frames (models). Moreover, we used the notion of neighborhood in three ways. First, we spoke about the class of all neighborhood structures (n2 -frames). Second, we made references to neighborhoods in the standard topological sense. Third, we used those topological neighborhoods to transform multi-topological frame into certain specific $\mathbf{n} \mathbf{2}$-frame. Hence, we shall repeat the most important things and sum up our considerations.

In section 3 we have described neighborhood semantics for intuitionistic modal logic. It is based on the notions of minimal ("intuitionistic") and maximal ("modal") neighborhoods.

In section 5 we have introduced $\mathbf{m t D}$-frames (models). They are collections of topological spaces. These spaces can intersect or form unions. We assumed that each space $\langle T, \tau\rangle$ has certain distinguished open set $D^{\tau}$. Then we have shown how it is possible to treat $\mathbf{n} \mathbf{2}$-frames as $\mathbf{m t D}$-frames. Shortly speaking, the main idea is to make connection between maximal (resp. minimal) neighborhoods and universes $T$ (resp. distinguished sets).

In section 7 we spoke about $\mathbf{t} \mathbf{2}$-frames (models). They are similar to the class of mtD but each topology is Alexandrov and we do not introduce distinguished sets anymore. We have shown how to transform those structures into neighborhood models. Let us repeat main steps of this reasoning. Assume that $W$ is the whole universe of a given $\mathbf{t} 2$-frame. Now let us take an arbitrary $w \in W$. For each topology $\tau$ we have minimal $\tau$-open $w$-neighborhood (because of Alexandrov property). We take intersection of all such minimal neighborhoods and treat it as $\bigcap \mathcal{N}_{w}$ (as the minimal $w$-neighborhood in the sense of $\mathbf{n} \mathbf{2}$-frames). Then we take intersection of all topological spaces to which $w$ belongs and this is our maximal neighborhood.

In section 8.1 we came back to $\mathbf{n} \mathbf{2}$-frames but we introduced another topology in those structures (different than in section 5). It is possible to transform $\mathbf{n} 2$-models with this topology into $\mathbf{t 3}$-multi-topological models which are based on $\mathbf{t} \mathbf{2}$-frames but with different valuation than in section 7 . 


\section{References}

[1] M. Božic, K. Došen, Models for normal intuitionistic modal logics, Studia Logica, Vol. XLIII (1984).

[2] Á. Császár, Generalized topology, generalized continuity, Acta Mathematica Hungarica, Vol. 96, No. 4 (2002), pp. 351-357.

[3] M. J. Collinson, B. P. Hilken, D. E. Rydeheard, An adjoint construction for topological models of intuitionistic modal logic. Extended abstract, http://sierra.nmsu.edu/morandi/old\%20files/TbilisiConference/ Collinson.pdf

[4] J. M. Davoren, Topological Semantics and Bisimulations for Intuitionistic Modal Logics and Their Classical Companion Logics, Logical Foundations of Computer Science 2007, Springer 2007.

[5] J. M. Davoren, V. Coulthard, T. Moor, R. P. Goré, A. Nerode, On Intuitionistic Modal and Tense Logics and Their Classical Companion Logics: Topological Semantics and Bisimulations, Annals of Pure and Applied Logic, Vol. 161 (2009), pp. 349-367.

[6] J. M. Davoren, V. Coulthard, T. Moor, R. P. Goré, A. Nerode, Topological semantics for Intuitionistic modal logics and spatial discretisation by $A / D$ maps, [in:] Workshop on Intuitionistic Modal Logic and Applications (IMLA), Copenhagen, Denmark 2002.

[7] D. de Jongh, F. Sh. Maleki, Two neighborhood semantics for subintuitionistic logics, http://events.illc.uva.nl/Tbilisi/Tbilisi2017/uploaded_ files/inlineitem/Dick_de_Jongh_Fateme_Shirmohammadzadeh_Maleki. pdf

[8] D. de Jongh, F. Sh. Maleki, Weak subintuitionistic logics, https://www. illc.uva.nl/Research/Publications/Reports/PP-2016-12.text.pdf

[9] M. Moniri, F. S. Maleki, Neighborhood semantics for basic and intuitionistic logic, Logic and Logical Philosophy, Vol. 23 (2015), pp. 339-355.

[10] E. Pacuit, Neighborhood Semantics for Modal Logic, Springer International Publishing AG 2017.

[11] V. H. Sotirov, Modal Theories with Intuitionistic Logic, [in:] Mathematical Logic, Proceedings of the Conference on Mathematical Logic, Dedicated to the Memory of A. A. Markov (1903-1979), September 22-23, 1980, pp. 139-171, Sofia 1984.

[12] T. Speer, A Short Study of Alexandroff Spaces, https://arxiv.org/pdf/ $0708.2136 . p d f$ 
[13] T. Witczak, Generalized Topological Semantics for Weak Modal Logics, https://arxiv.org/pdf/1904.06099.pdf.

[14] T. Witczak, Intuitionistic Modal Logic Based on Neighborhood Semantics Without Superset Axiom, https://arxiv.org/pdf/1707.03859.pdf.

Institute of Mathematics

University of Silesia

Bankowa 14

40-007 Katowice, Poland

e-mail: tm.witczak@gmail.com 